\title{
Trio Constrained Adaptive Noise Removal (TCANR) Mechanism for Salt and Pepper Noise in Image Classification
}

\author{
G Muthu Krishnan ${ }^{1}$, Capt.Dr.S.Santhosh Baboo ${ }^{2}$ \\ Research Scholar, Dravidian University, Kuppam ${ }^{1}$ \\ Associate Professor, P.G. \& Research Department of Computer science, D.G. Vaishnav College, Chennai ${ }^{2}$
}

\begin{abstract}
Noise removal (also called as denoising) of an image is a vital task in multi-class image classification. Three major shortcomings in Weighted Nuclear Norm Minimization (WNNM) are identified. Firstly, WNNM's patch matching based on the noisy data will considerably augment the risk of patch mismatching. This shortcoming is overcome by performing the grouping task based on noise contentment. Secondly, the fixed feedback percentage which keeps on feeds back ten percent of the residual image to the next iteration despite the consequences of noise levels. This shortcoming is ruled out by incorporating relative feedback mechanism. Finally, the unchanged / constant number of iterations for different noise not considering the distinctions in image content that which will certainly fails to deem the degree of detail in the image. For this variable termination criterion is used. The proposed work is named as Trio Constrained Adaptive Noise Removal (TCANR). Performance metric peak signal to noise ratio (PSNR) is chosen. Four existing methods are taken into account for comparing the proposed TCANR. Extensive simulations are conducted using MATLAB and the results prove that the proposed TCANR performs better in terms of PSNR when compared with the existing methods.
\end{abstract}

Keywords: WNNM, Classification, Multi-label, Noise Removal, Quality, Denoise, PSNR, TCANR, TV, FBF, LLSure, LAPB, Corel 5k, IAPR-TC12, PASCAL-VOC-2007, PASCAL-VOC-2010

\section{INTRODUCTION}

Classification of multi-label image is more muddled than the classification of single-label image because of the accompanying difficulties. Firstly, closer view protests in multi-label images are not adjusted as in single-label images. In few multi-label images, critiques are situated at unusual points with various range and postures. Surprisingly more dreadful, some closer view items are impeded by others. In spite of the difficulties, there are likewise extra prompts that could be used to help multilabel image classification.

Noise removal is one of the primary tasks in image processing and particularly in multi-label image classification. The nature of a computerized image always gets weakens by the impulse noise in the record or transmission. Images are definitely undermined by motive noise, brought about by failing pixels in camera sensors, accuse memory areas in equipment, diffusion in a filter which are noisy, and bit mistakes during transmission. There are two sorts of motive (or impulse) noise, which are random valued and salt-and-pepper noises. Salt-andpepper noise can truly degenerate the images where the undermined pixel takes either the greatest or the lowest level of gray. This noise can altogether fall apart the nature of an image. The strategy of expelling this kind of impulse noise is capably a critical research task. Impulse noise is a choice like "on-off" that influences the image all of a sudden. Impulse noise is created at the period of receiving images through sensors, in the midst of the transmission of images or because of the barometrical varieties, for

instance, lightening etc. At the point when an image is ruined due to the impulse noise, the nature of the image is corrupted at a substantial degree. Thus, the expulsion of impulse noise from the caught image is important to upgrade the nature of the image. Force of impulse noise has the inclination of being either moderately maximum or minimum; consequently, aspect of the image is extremely influenced because of high frequency of impulse noise. Safeguarding the image elements and weakening of noise are the two critical parts of image rebuilding. Overall, the linear filters are viable for added substance noises. In any case, their execution for evacuation of impulse noise will not be satisfactory. In this manner, non-linear filters are favored for expulsion of impulse noise. In this phase of research work, removal of salt and pepper noise is obtained. The proposed research work contributes mainly with the objective of removal of salt and pepper noise. Another important objective of the proposed research work is to reduce the peak signal to noise ratio when compared with the existing denoising algorithms. The next section presents the related works.

\section{LITERATURE REVIEW}

Xianquan Zhang et al.,2015 proposed a basic and proficient rebuilding calculation with the hypothesis of image inpainting. It takes the input as noisy pixels as missing the information for inpainting, attentively chooses complexity cover regarding points of interest of nearby region, and accomplishes rebuilding by repetitive complex 
process. Luis González Jaime et al.,2014 presented an approach in view of dealing with obscure noise models. To carry out this, distinctive refined images were acquired, and then it was consolidated utilizing multifuzzy sets and averaging total capacities. With a specific end goal to handle clashing issues of noise smoothing and depth protection, Muhammad Habib et al.,2016 proposed an approach by utilizing versatile fuzzy surmising framework for irregular esteemed impulse noise identification and evacuation, where its filter utilizes the strength based directional insights to develop versatile fuzzy enrollment capacities which assumes an essential part in fuzzy intruding framework.

V.P. Ananthi and P. Balasubramaniam.,2016 proposed a technique which explores image denoising by demonstrating this unclearness as deterioration. An IVIFS for an image was created by limiting entropy. At L/that point sort diminished IVIFS was acquired by taking probabilistic value of the enrolled interim. At long last, uproarious pixels were identified by utilizing the directional pieces and were filtered by utilizing fuzzy filter. Lianghai Jin et al.,2016 proposed a way to deal with impulse noise evacuation in shading images, where arrangement was a quaternion exchanging vector filter in which the impulse recognition comprises of more than one phase. Amarjit Roy et al.,2016 proposed a support vector machine (SVM) classification based Fuzzy filter (FF) for expulsion of impulse noise from dark scale images. At the point when an image was influenced by impulse noise, the nature of the image was contorted since the homogeneity among the pixels was broken. SVM was joined for identification of impulse noise from images.

Guorong Gao et al.,2015 presented two-phase denoising technique for the expulsion of arbitrary esteemed impulse noise (RVIN) in images. The principal phase of its calculation applies a impulse noise recognition schedule which was a refinement of the HEIND calculation and it was exceptionally precise in distinguishing the area of the noisy pixels, where its end phase was a image inpainting schedule that wass intended to reestablish the missing data at those pixels that have been distinguished amid the principal phase. Amarjit Roy and Rabul Hussain Laskar.,2016 proposed a multiclass support vector machine (SVM) based versatile filter for expulsion of impulse noise from shading images. The nature of the image gets corrupted because of the nearness of noise by impulse. Subsequently, the homogeneity among the pixels gets bended which should be reestablished. Examination of Ching-Ta Lu et al.,2016 exhibited a three-valuesweighted technique for the expulsion of salt-and-pepper noise. At first, a variable-estimate neighborhood window was utilized to break down every outrageous pixel.

The target of a approach developed by Bin Zhou et al.,2012 was to diffuse the different values when measured in different direction and exclude the noise. Numerous methods were proposed for randomly varied models. The characteristics of the diffusion were controlled by nonlinear partial differential equation. Jian Lu et al.,2016 proposed a variety representation for rebuilding of images ruined by multiplicative noise, where it demonstration were figured in the logarithm transform space of the attractive images comprises of an data desirable style a quadratic style and an aggregate variety regularizer. In Xiang Guo et al.,2017 stationary wavelet transform (SWT) based technique was proposed to denoise the advanced image with the low noise, and the SWT denoising calculation was displayed after the investigating of the light noise.

Amarjit Roy and Rabul Hussain Laskar.,2017 proposed a non-causal linear prediction based adaptive vector median filter for removal of high density impulse noise from color images. In the proposed method, if the pixel under operation was found to be corrupted, the filtering operation will be carried out. In Sasan Mahmoodi.,2017 a nonlinear method based on anisotropic diffusion notion was proposed to remove noise from noisy signals modulated with multiple carrier signals by preserving carrier signals as well as discontinuities present in the original noiseless signals. The next section elaborates on the proposed research work with certain technical background and preliminaries.

\section{TRIO CONSTRAINED ADAPTIVE NOISE REMOVAL (TCANR) MECHANISM FOR MULTI - LABEL IMAGES}

\subsection{Technical Background and Preliminaries}

Weighted Nuclear Norm Minimization uses broadneighbor ascetic-resemblance to concentrate associated area of a given reference into a grid to structure a grouping. Heaps of strategies have been created to play out the grouping function. In undeniable reality, a simple and adequate grouping strategy is made use with the assistance of WNNM and it is to be functioned by pairwise ascertaining the closeness between the reference area and hopeful ones arranged at various spatial areas. It is critical that the closeness between two areas is enumerated by making utilization of the evaluation of more than a space. Minor parting means lifted closeness to resemblance. A blend of separation measures are most likely be utilized and the WNNM strategy utilizes the Euclidean separation measure for figuring the same. The resemblance between a indication area $\mathbf{P}_{f}$ of mass $n \times n$ and contestant $P_{i}$ can be showed as:

$$
\operatorname{Sim}\left(P_{f}, P_{i}\right)=\frac{\left\|P_{f}-P_{i}\right\|_{F}^{2}}{n^{2}} \ldots
$$

The WNNM mechanism arranges in descending order of all the similar patches based on the similarity values and then gathers the opening $N$ patches as the similar patches that will form a matrix. In order to obtain a noise - free patch, nuclear norm minimization (NNM) is used as its convex respite, and is formulated as:

$$
X j=\arg \min _{X_{j}}\left\|Y_{j}-X_{j}\right\|_{F}^{2}+\lambda\left\|X_{j}\right\|_{*} \ldots
$$


where $\left\|X_{j}\right\|_{*}$ the nuclear norm of is $X_{j}, Y_{j}$ is the observation matrix, and $\lambda$ is a positive constant.

For shrinking every singular value with the same amount $\lambda$ WNNM modeled a weighted nuclear norm of matrix $X_{j}$, by the below equation

$$
\left\|X_{j}\right\|_{w, *}=\sum_{i}\left|w_{i} \sigma_{i}\left(X_{j}\right)\right| \ldots
$$

where $W=\left[w_{1}, w_{2}, \ldots w_{n}\right]$ and $w_{i}$ is the non-negative weighted coefficient, and $\sigma_{i}\left(X_{j}\right)$ is the $i$-th singular value of $X_{j}$. Hence Eq. (2) gets rewritten as:

$$
X_{j}=\arg \min _{x_{j}} \frac{1}{\sigma_{n}^{2}}\left\|Y_{j}-X_{j}\right\|_{F}^{2}+\left\|X_{j}\right\|_{w, *}
$$

Into the bargain, WNNM takes advantage of an iterative regularization technique that will nourish partial method noise back to the next denoising process which results in elevated denoising performance.

\subsection{Proposed Work}

First three important shortcomings in WNNM have been identified. First shortcoming is, WNNM's patch matching based on the noisy data that will considerably augment the risk of patch mismatching. Second shortcoming is the fixed feedback percentage which keeps on feeds back ten percent of the residual image to the next iteration despite the consequences of noise levels. Third shortcoming is the unchanged / constant number of iterations for different noise not considering the distinctions in image content that which will certainly fails to deem the degree of detail in the image.

\subsubsection{Grouping based on Noise Containment}

For overcoming the first shortcoming of WNNM, this subsection presents the mechanism called grouping based on noise containment. Let $P_{f}$ be a reference patch of size $n \times n$ in a noisy image, and $P_{i}$ be a candidate similar patch. Here we only consider the additive Gaussian noise. Hence, $P_{j}$ and $P_{i}$ can be written as:

$$
\left\{\begin{array}{l}
P_{f}=P_{f}^{0}+N_{f}^{o} \\
P_{i}=P_{i}^{0}+N_{i}^{0}
\end{array}\right.
$$

where $P_{f}^{0}$ and $P_{i}^{o}$ are the latent clean patches of $P_{f}$ and $P_{i}$ respectively, and $N_{f}^{o}$ and $N_{i}^{o}$ are the noise with mean 0 and standard deviation $\sigma$. Pertaining to clean images and noise are uncorrelated, the mathematical representation will be:

$$
E\left\{\operatorname{Sim}\left(P_{f}, P_{i}\right)\right\} \approx \operatorname{Sim}\left(P_{f}^{0}, P_{i}^{o}\right)+\sigma^{2}
$$

From the equation (6), it is observed that the similarity measure of noisy patches is a biased estimator when compared to clean ones which will categorize a dissimilar patch into the similar patch group by fault. For overcoming this pitfall and to restrain the noise effect on the matching of similar patches, a coarse prefiltering is used by using Discrete Cosine Transform (DCT).

\subsubsection{Relative Feedback Mechanism}

For overcoming the second shortcoming of WNNM, this sub-section presents the mechanism called relative feedback mechanism. Let $Y$ be an original noisy image and $J$ be a denoising operator. The denoised image outcome $\hat{X}$ will be:

$$
\hat{X}=J(Y) \text {.. }
$$

Hence the noise $\Delta X$ will be,

$$
\Delta X=Y-\hat{X}
$$

The relative feedback mechanism is performed using the below formula

$$
f_{n}(i)=\frac{255 *\left(f(i)-f_{\min }\right)}{f_{\text {max }}-f_{\text {min }}} .
$$

By taking into account of salt-and-pepper noise model the evaluation of the denoising performance via method noise is evaluated. If truth be told, it is impracticable to have clean images while performing the denoising process.

\subsubsection{Variable Termination Criterion}

For overcoming the third shortcoming of WNNM, this sub-section presents the mechanism called variable termination criterion. Based on the noise inducted in the image and quality of the image, it is imperative to resolve the number of iterations. Hence the correlation measure is carried out:

$$
\operatorname{correl}(k)=\operatorname{corr}\left(H . * \hat{X}^{(k)}, H . *\left(Y-\hat{X}^{(k)}\right)\right) \ldots
$$

where $H$ is a matrix that indicates the active regions of an image, and $\hat{X}^{(k)}$ is the denoised image after $k$ iterations. $H$ is obtained by dilating the result of edge detection $\hat{X}(1)$. So $H . * \hat{X}^{(k)}$ and $H . *(Y-\hat{X})^{(k)}$ are the active regions of the $k$-th iteration denoised image and the method-noise image, respectively. The best iteration is the one that hits the minimum absolute value in the 'correl' vector, which is used as the terminating criterion.

The proposed TCANR algorithm is portrayed below: 


\section{Algorithm 1 Image Denoising with Trio Constrained Adaptive Noise Removal}

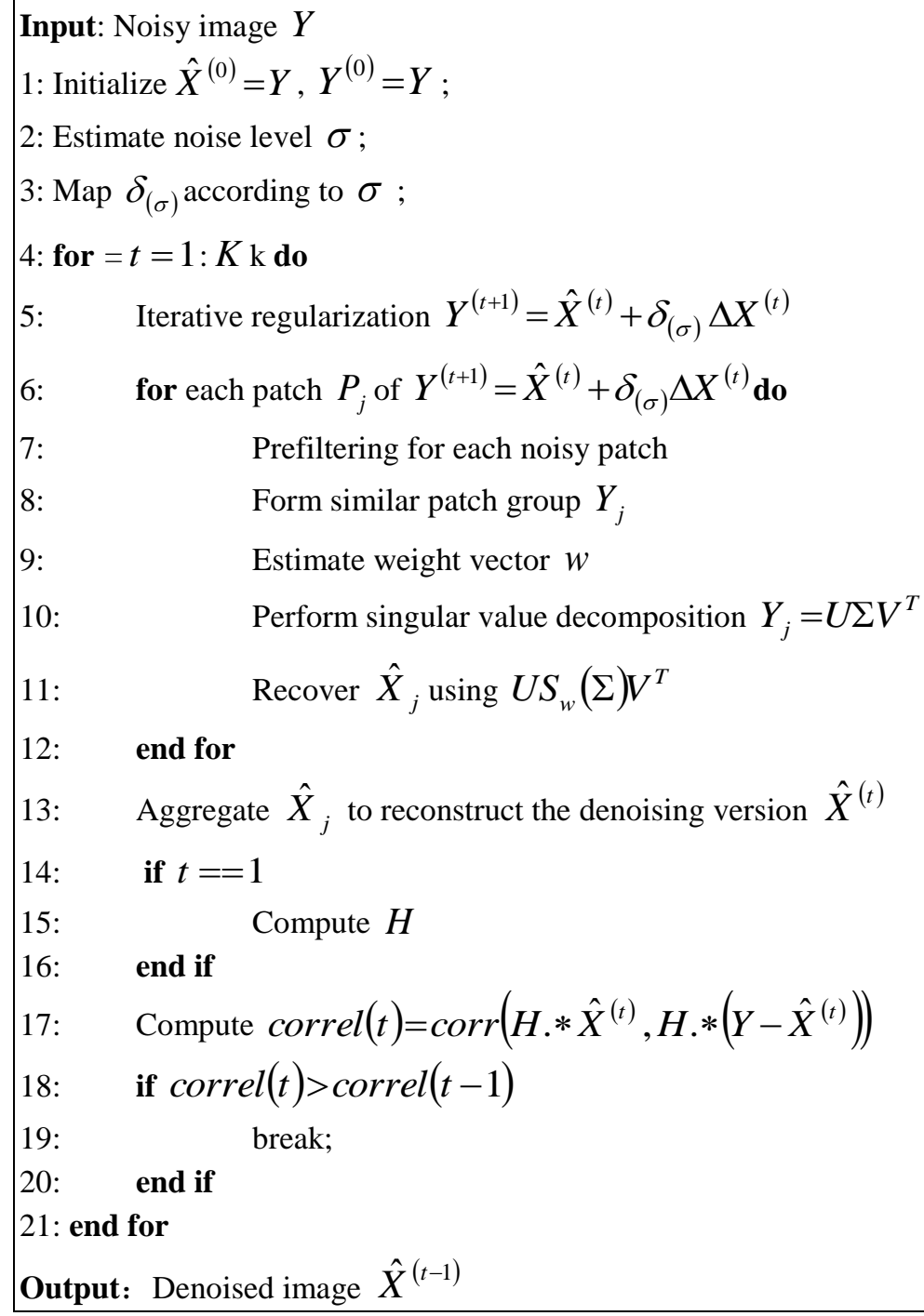

Output: Denoised image $\hat{X}^{(t-1)}$

The next section explains the results and discussions of the proposed method named Trio Constrained Adaptive Noise Removal (TCANR).

\section{RESULTS AND DISCUSSION}

The performance of this TCANR is compared with 4 methods (Total Variation (TV) Method [L. Rudin et al.,1992], Fast Bilateral Filter (FBF) Method [Q. Yang et al.,2009], LL Sure (LLSure) Method [T. Qiu et al.,2013], The obtained original images of Corel5k dataset are shown Locally Adaptive Patch - Based (LAPB) Method in the Fig.01. Then salt and pepper noise is added to the [Minyoung Kim.,2015]) over four datasets (Corel 5k images of Corel5k dataset is shown in Fig.02. Then the Dataset [P. Duygulu et al.,2002], IAPR - TC12 Dataset denoised result image of the proposed TCANR is [M. Grubinger.,2007], PASCAL-VOC-2007 Dataset [M. presented for the Corel5k dataset in Fig.03. The PSNR Everingham et al.,2007], PASCAL-VOC-2010 Dataset comparison for the dataset is presented in the Fig.04. The [M. Everingham et al.,2010]). In this research work 10 images are taken from each of the four datasets.

\subsection{Corel 5k Dataset}

Corel-5K [35] is widely used for testing methods for image retrieval and annotation. The dataset is comprised of concepts manually annotated for about 5000 images. The concept keywords are mostly the object category names. The dataset provides the fixed splits of training/test sets, roughly taking $90 \% / 10 \%$ proportions of the entire samples.

PSNR values for the Corel57 dataset is presented in the Table 1. From the results inferences made is, the maximum noise ratio for the dataset Corel5k is $19.2 \mathrm{~dB}$ and the minimum noise ratio is $14.7 \mathrm{~dB}$. This projects the improved performance of TCANR in Corel5k dataset. 
International Journal of Advanced Research in Computer and Communication Engineering ISO 3297:2007 Certified

Vol. 6, Issue 3, March 2017

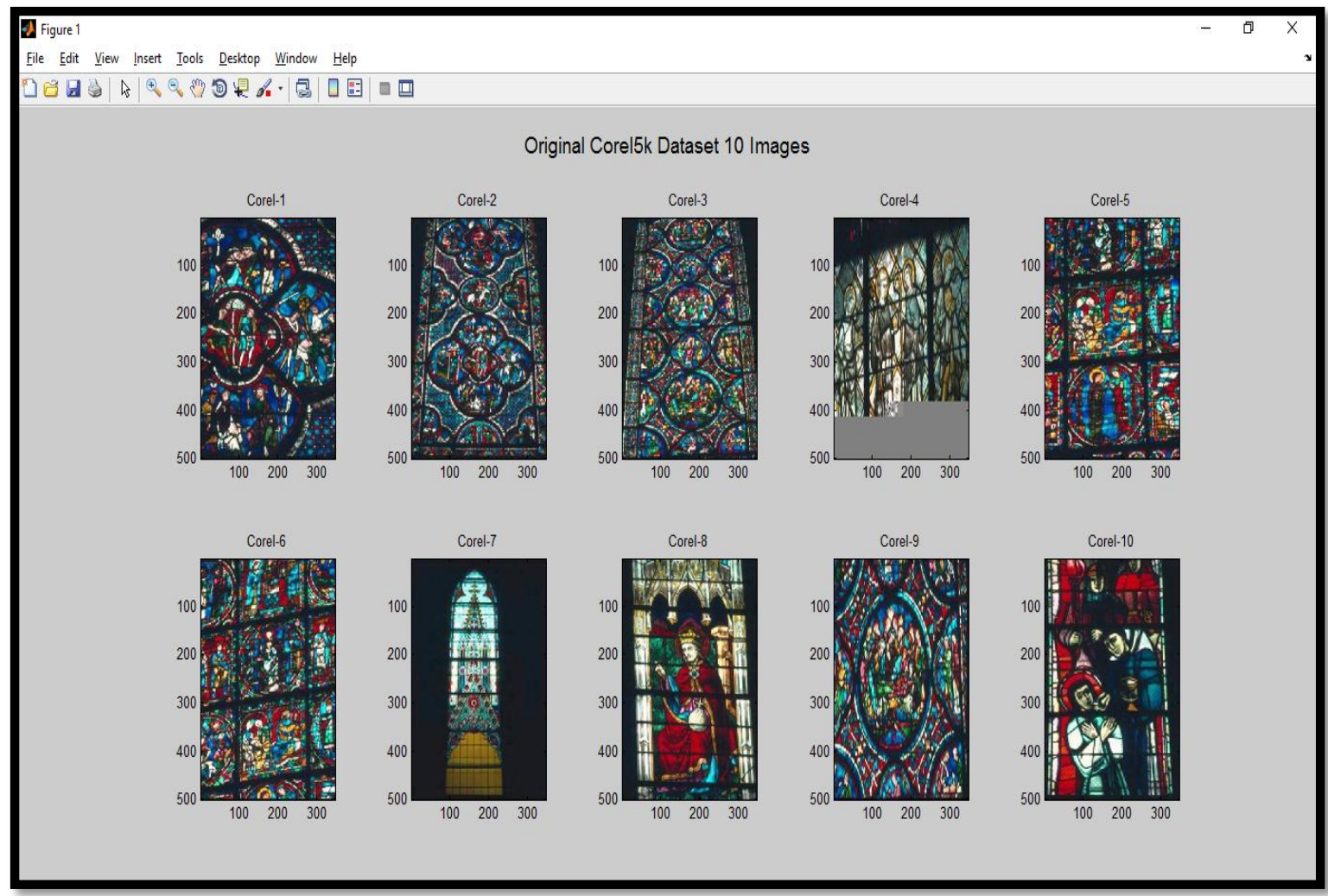

Fig. 01. Original Image of Corel 5K Dataset

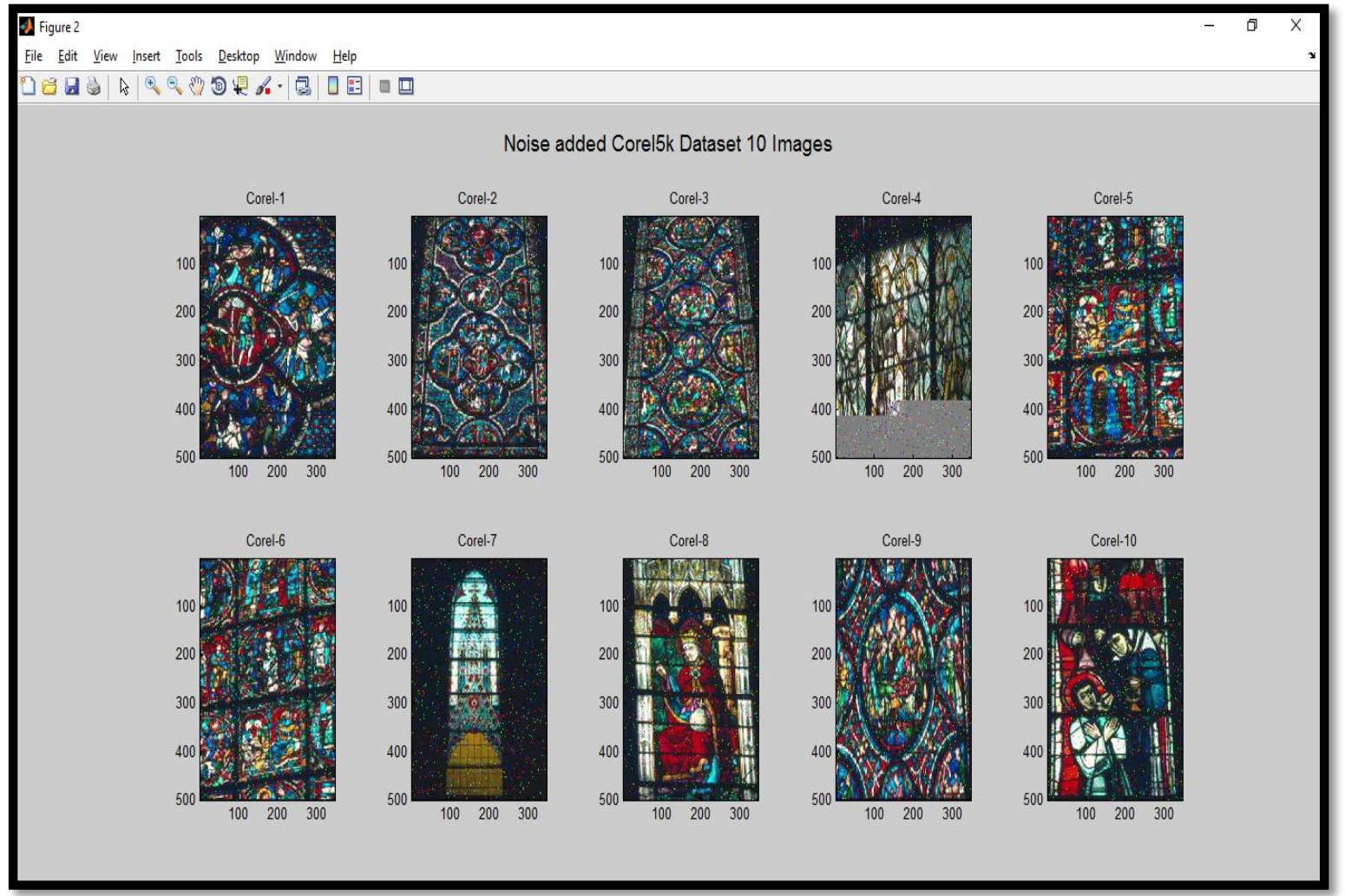

Fig. 02. Noise Added Image of Corel 5K Dataset 
International Journal of Advanced Research in Computer and Communication Engineering ISO 3297:2007 Certified

Vol. 6, Issue 3, March 2017

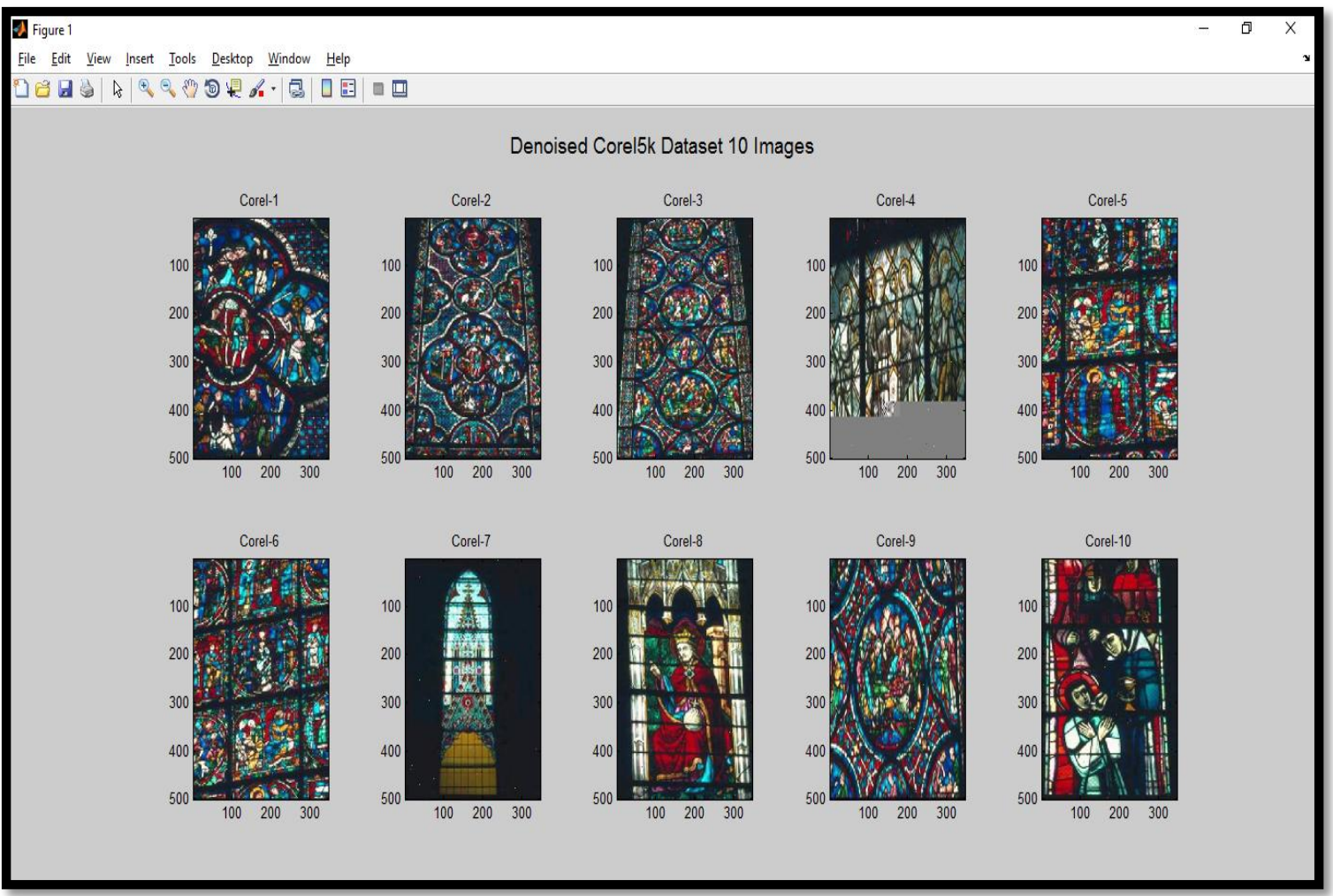

Fig. 03. Denoised Image for Corel 5K Dataset

Table 01. PSNR Comparison for the Corel 5K Dataset

\begin{tabular}{|c|c|c|c|c|c|}
\hline \multirow{2}{*}{ Image Title } & \multicolumn{5}{|c|}{ PSNR in dB } \\
\cline { 2 - 6 } & TV [\#] & FBF [@] & LLSure [\&] & LAPB [*] & TCANR \\
\hline Corel - 01 & 30.1 & 27.9 & 24.2 & 18.1 & 17.5 \\
\hline Corel - 02 & 34.0 & 26.6 & 23.1 & 23.8 & 16.5 \\
\hline Corel - 03 & 28.7 & 27.9 & 26.3 & 20.0 & 16.6 \\
\hline Corel - 04 & 33.6 & 30.6 & 26.5 & 22.6 & 17.6 \\
\hline Corel - 05 & 33.2 & 27.7 & 22.1 & 23.6 & 16.8 \\
\hline Corel - 06 & 34.1 & 28.7 & 26.9 & 24.1 & 19.2 \\
\hline Corel - 07 & 32.1 & 27.0 & 25.7 & 22.3 & 19.0 \\
\hline Corel - 08 & 33.8 & 27.4 & 25.1 & 24.8 & 18.7 \\
\hline Corel - 09 & 28.6 & 25.3 & 22.9 & 18.9 & 14.7 \\
\hline Corel - 10 & 31.8 & 29.9 & 23.7 & 20.9 & 18.6 \\
\hline
\end{tabular}

[where \# $\rightarrow$ L. Rudin et al.,1992, @ $\rightarrow$ Q. Yang et al.,2009, $\& \rightarrow$ T. Qiu et al.,2013, $\rightarrow$ Minyoung Kim.,2015] 


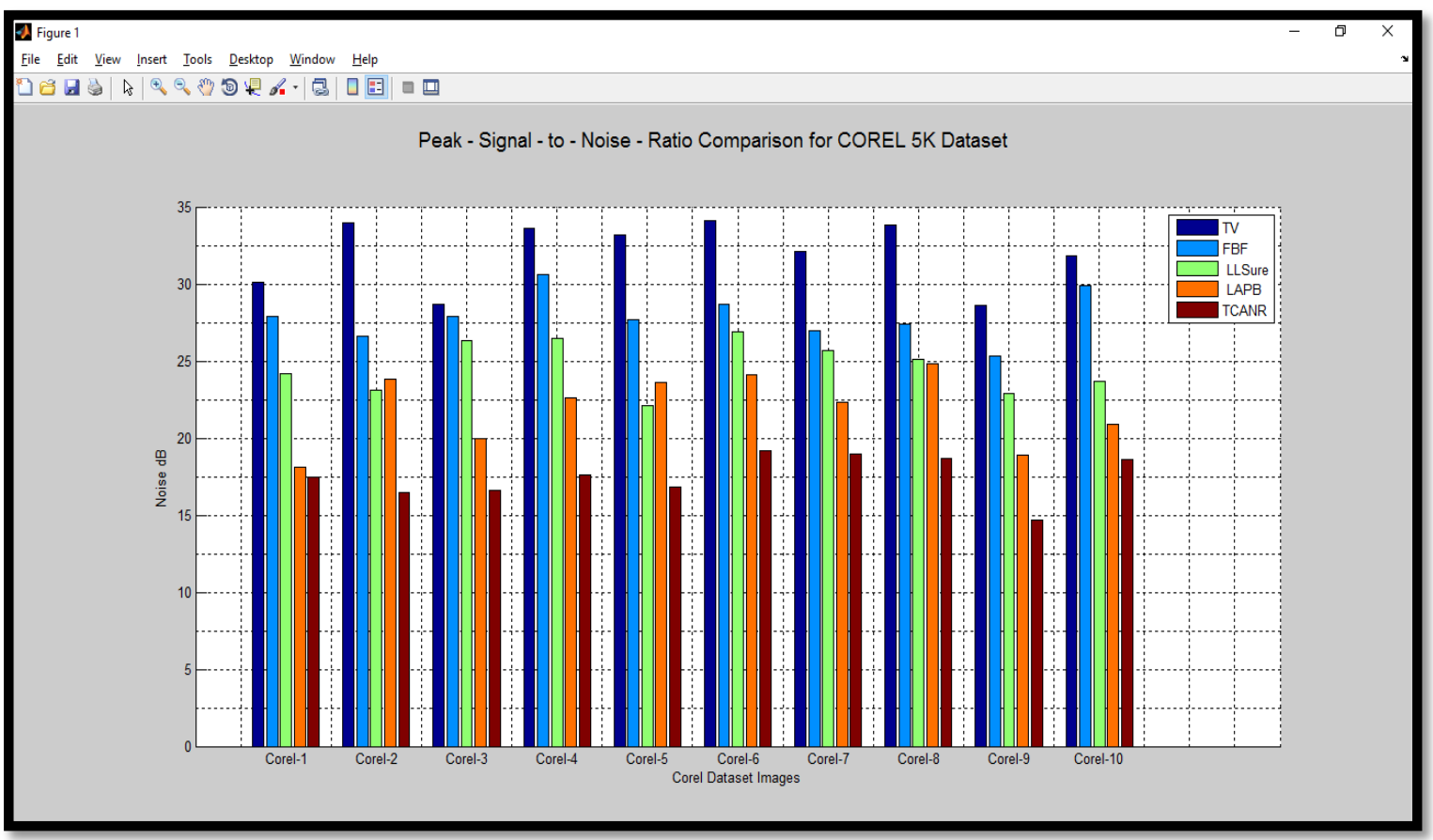

Fig. 04. Matlab Result - PSNR Comparison for COREL 5K Dataset

\subsection{IAPR - TC12 Dataset}

The image collection of the IAPR TC-12 consists of Then the denoised result image of the proposed TCANR is 20,000 images taken from locations around the world and presented for the IAPR - TC12 dataset in Fig.07. The comprising an assorted cross-section of images. This PSNR comparison for the dataset is presented in the includes pictures of different sports and actions, Fig.08. The PSNR values for the each of the four datasets photographs of people, animals, cities, landscapes and are presented in the Table 2. From the results inferences many other aspects of contemporary life. made is, the maximum noise ratio for the dataset IAPR The obtained original images of IAPR - TC12 dataset are TC-12 is $17.3 \mathrm{~dB}$ and the minimum noise ratio is $12.9 \mathrm{~dB}$. shown in the Fig.05. Then salt and pepper noise is added This projects the improved performance of TCANR in to the images of IAPR - TC12 dataset is shown in Fig.06. IAPR TC-12 dataset.

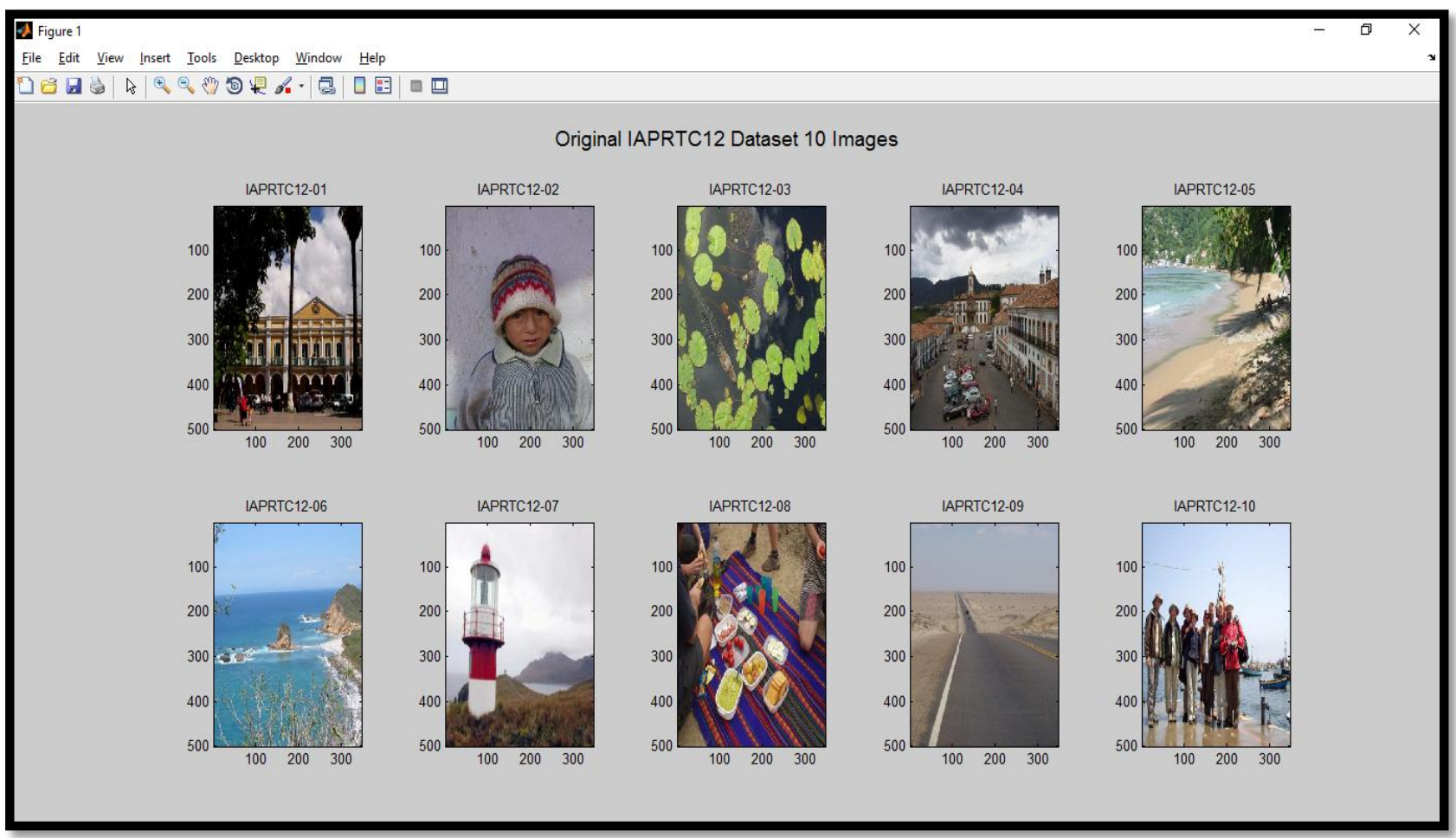

Fig. 05. Original Image of IAPR - TC12 Dataset 
International Journal of Advanced Research in Computer and Communication Engineering ISO 3297:2007 Certified

Vol. 6, Issue 3, March 2017

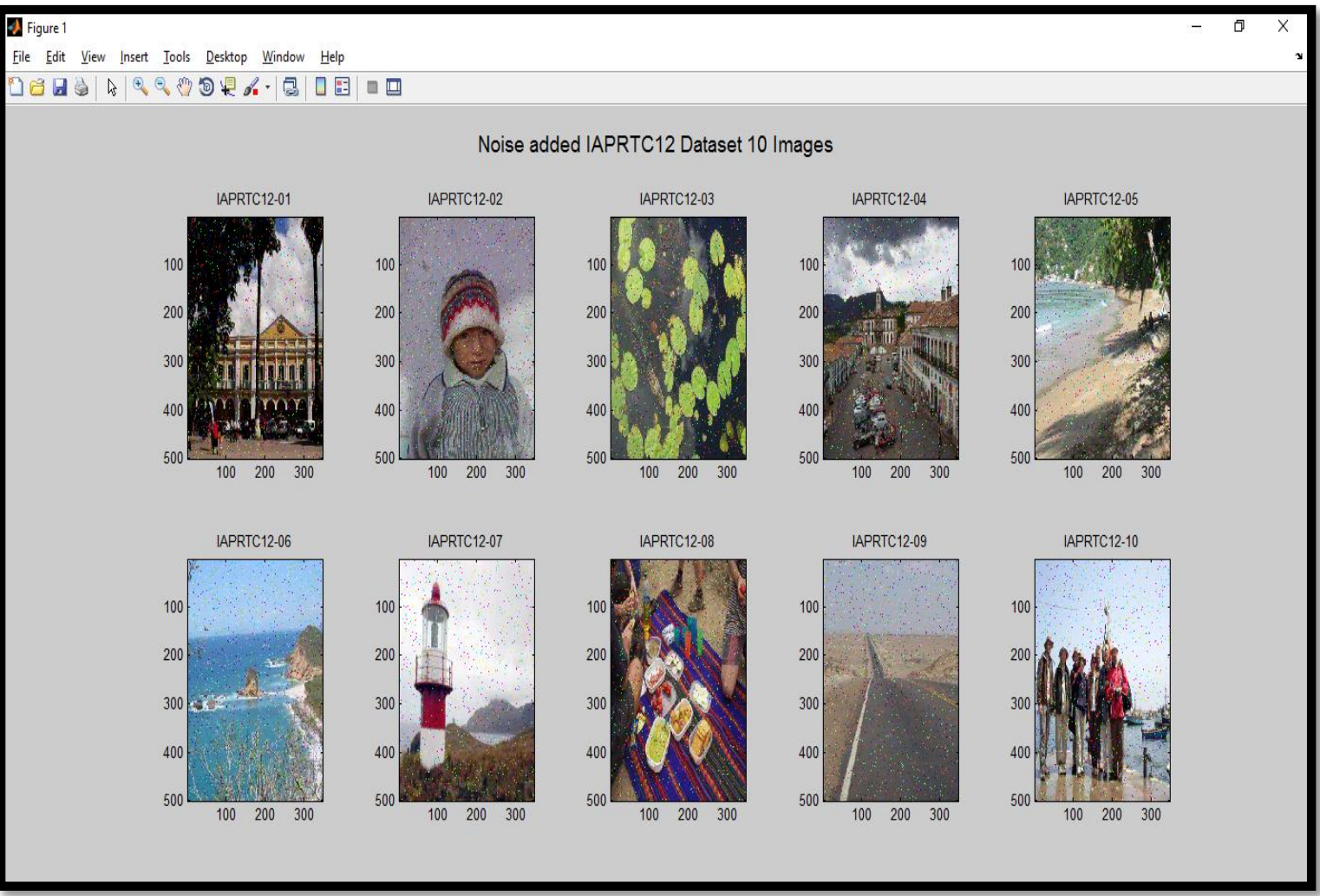

Fig. 06. Noise Added Image of IAPR - TC12 Dataset

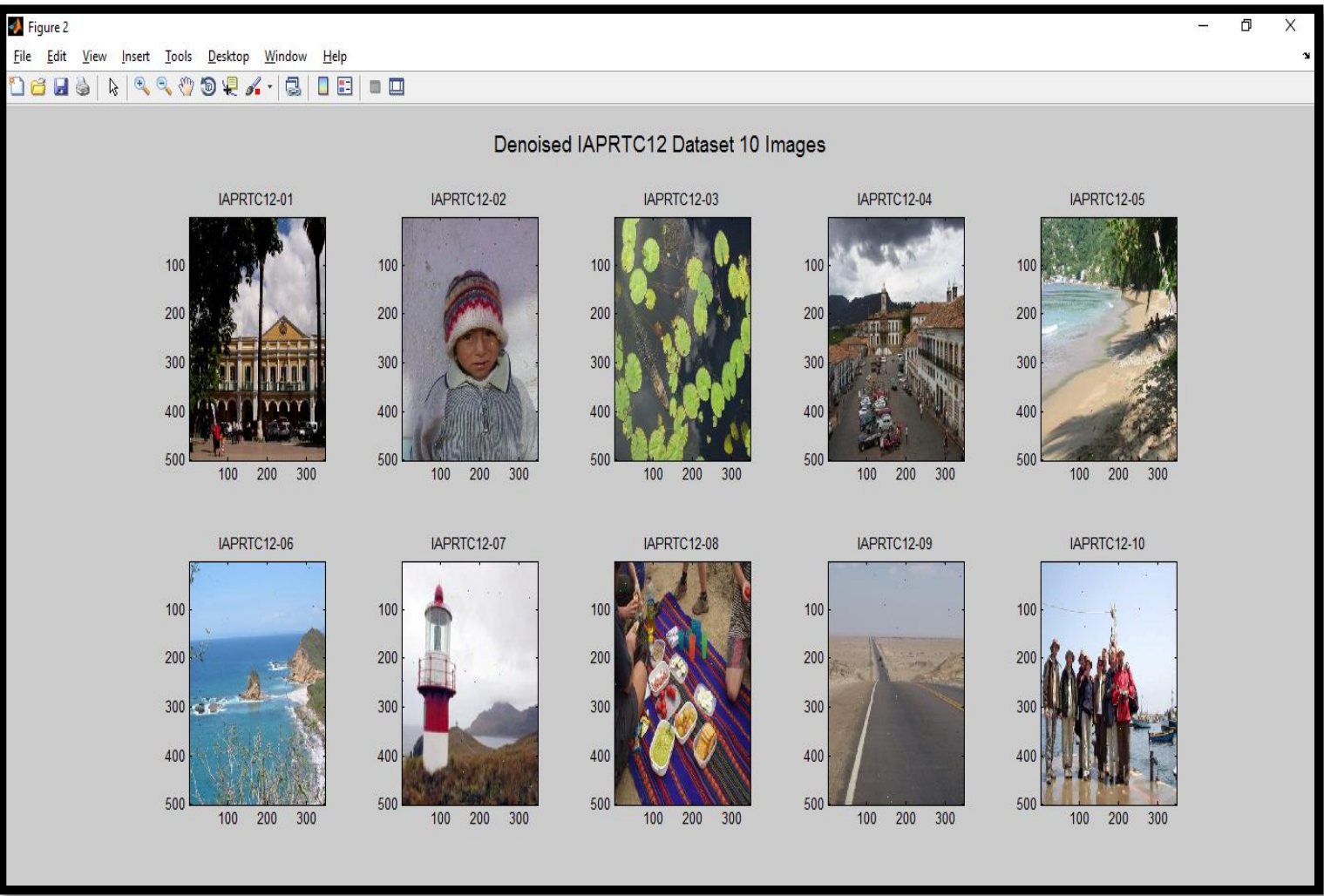

Fig. 07. Denoised Image for IAPR - TC12 Dataset 
International Journal of Advanced Research in Computer and Communication Engineering ISO 3297:2007 Certified

Vol. 6, Issue 3, March 2017

Table 02. PSNR Comparison for the IAPR - TC12 Dataset

\begin{tabular}{|c|c|c|c|c|c|}
\hline \multirow{2}{*}{ Image Title } & \multicolumn{5}{|c|}{ PSNR in dB } \\
\cline { 2 - 6 } & TV [\#] & FBF [@] & LLSure [\&] & LAPB [*] & TCANR \\
\hline IAPR - TC12 - 01 & 30.9 & 26.2 & 21.0 & 20.1 & 14.0 \\
\hline IAPR - TC12-02 & 32.0 & 26.9 & 21.6 & 17.6 & 15.1 \\
\hline IAPR - TC12-03 & 32.6 & 24.2 & 21.6 & 22.4 & 12.9 \\
\hline IAPR - TC12-04 & 32.1 & 24.3 & 25.3 & 18.6 & 17.1 \\
\hline IAPR - TC12-05 & 29.2 & 29.2 & 23.6 & 22.4 & 14.9 \\
\hline IAPR - TC12 - 06 & 31.7 & 24.4 & 22.5 & 22.7 & 15.7 \\
\hline IAPR - TC12-07 & 32.5 & 24.8 & 22.9 & 17.3 & 14.5 \\
\hline IAPR - TC12 - 08 & 32.5 & 28.1 & 23.9 & 19.1 & 17.2 \\
\hline IAPR - TC12-09 & 32.1 & 26.4 & 25.7 & 18.1 & 14.2 \\
\hline IAPR - TC12 - 10 & 29.5 & 27.8 & 23.0 & 17.4 & 17.3 \\
\hline
\end{tabular}

[where \# $\rightarrow$ L. Rudin et al.,1992, @ $\rightarrow$ Q. Yang et al.,2009, \& $\rightarrow$ T. Qiu et al.,2013, $\rightarrow$ Minyoung Kim.,2015]

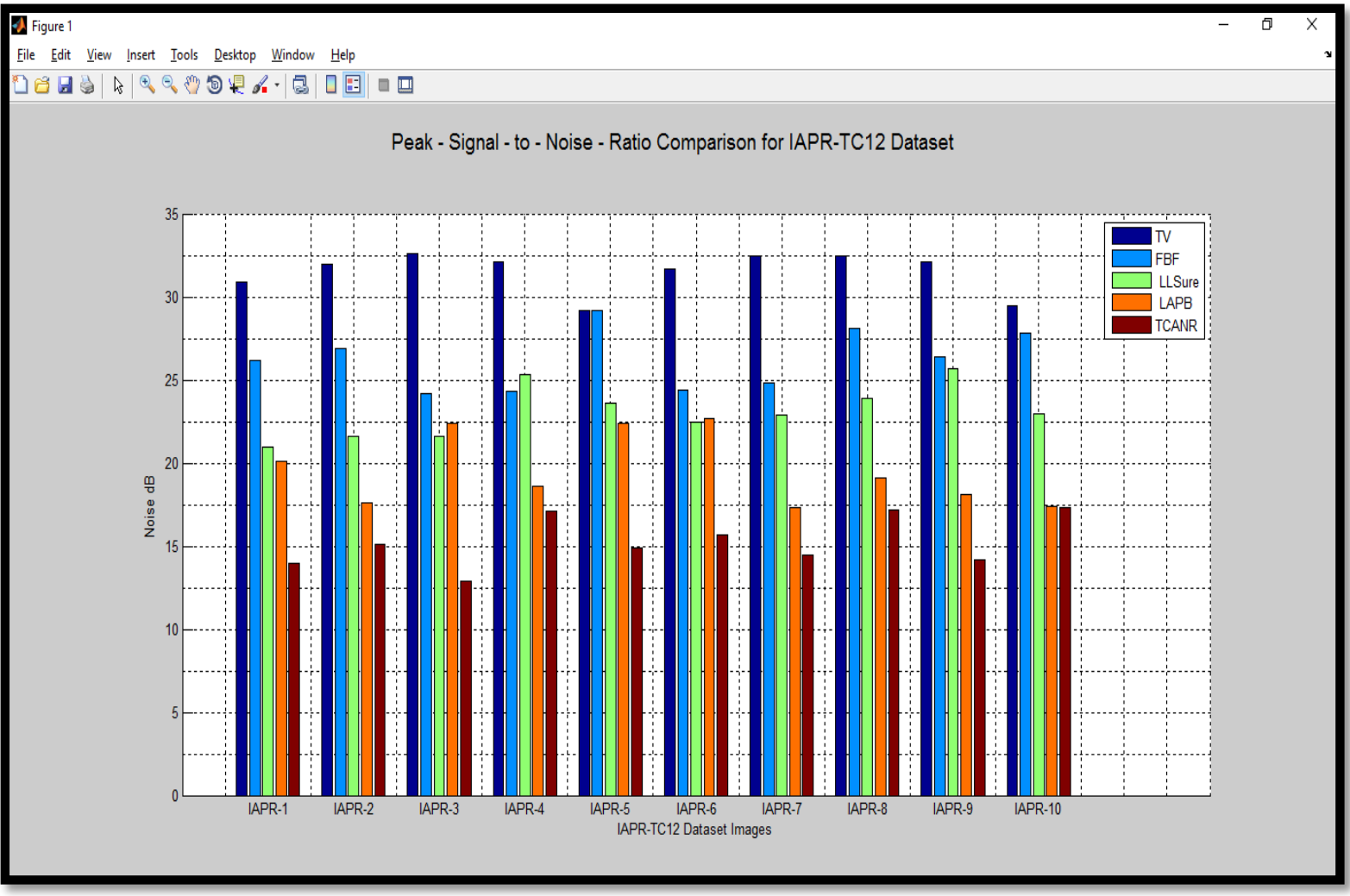

Fig. 08. Matlab Result - PSNR Comparison for IAPR - TC12 Dataset 


\subsection{PASCAL - VOC - 2007 Dataset}

The training data provided consists of a set of images; each image has an annotation file giving a bounding box and object class label for each object in one of the twenty classes present in the image. Note that multiple objects from multiple classes may be present in the same image. It contains 9963 images (VOC-2007) labeled with concepts including person, vehicle, dog, and so on.

The obtained original images of PASCAL-VOC-2007 dataset are shown in the Fig.09. Then salt and pepper

noise is added to the images of PASCAL-VOC-2007 dataset is shown in Fig.10. Then the denoised result image of the proposed TCANR is presented for the PASCALVOC-2007 dataset in Fig.11. The PSNR comparison for the dataset is presented in the Fig.12. The PSNR values for the each of the four datasets are presented in the Table 3. From the results inferences made is, the maximum noise the dataset PASCAL-VOC-2007 is $19.7 \mathrm{~dB}$ and the minimum noise ratio is $15.7 \mathrm{~dB}$. This projects the improved performance of TCANR in PASCAL-VOC2007 dataset.

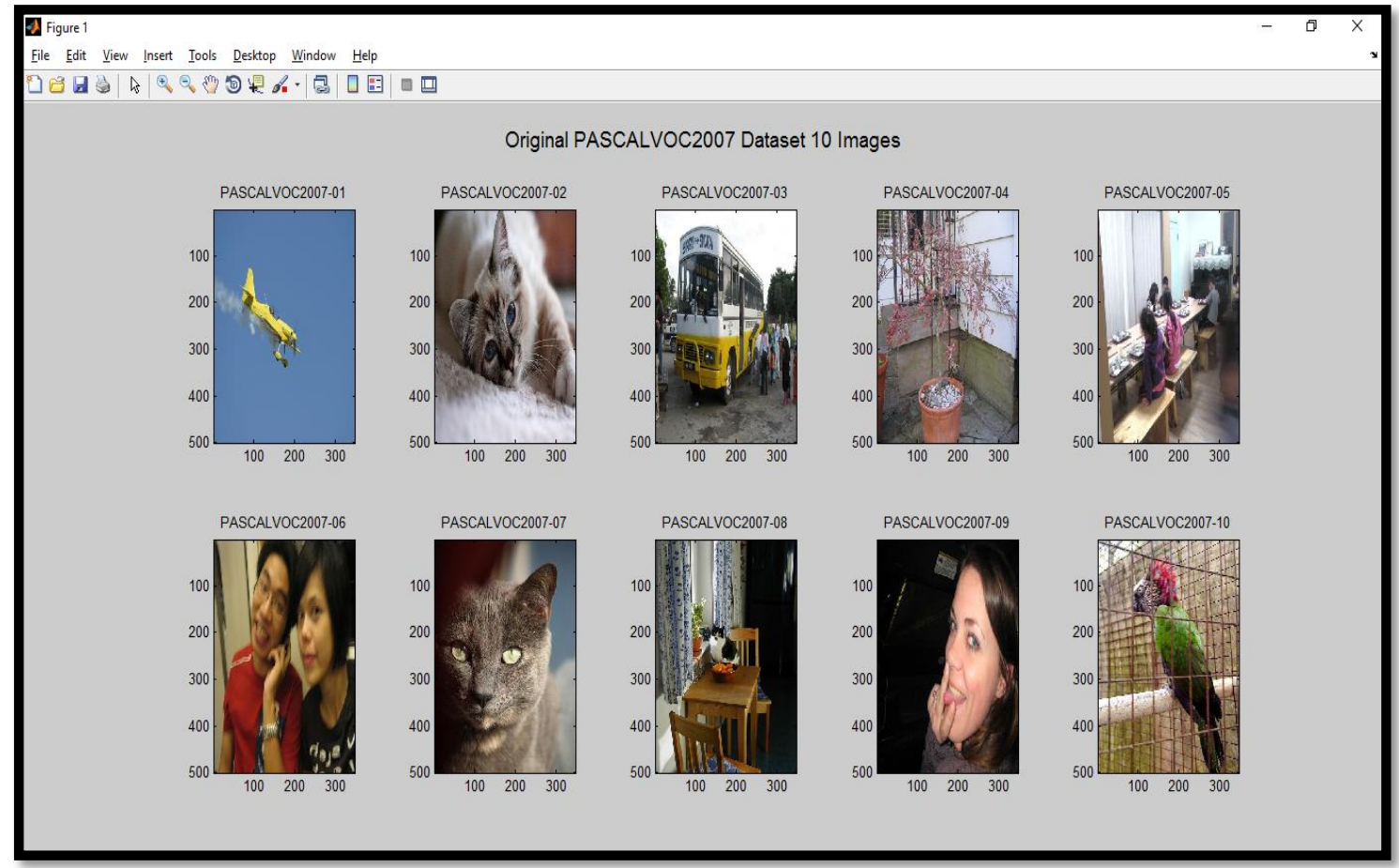

Fig. 09. Original Image of PASCAL - VOC - 2007 Dataset

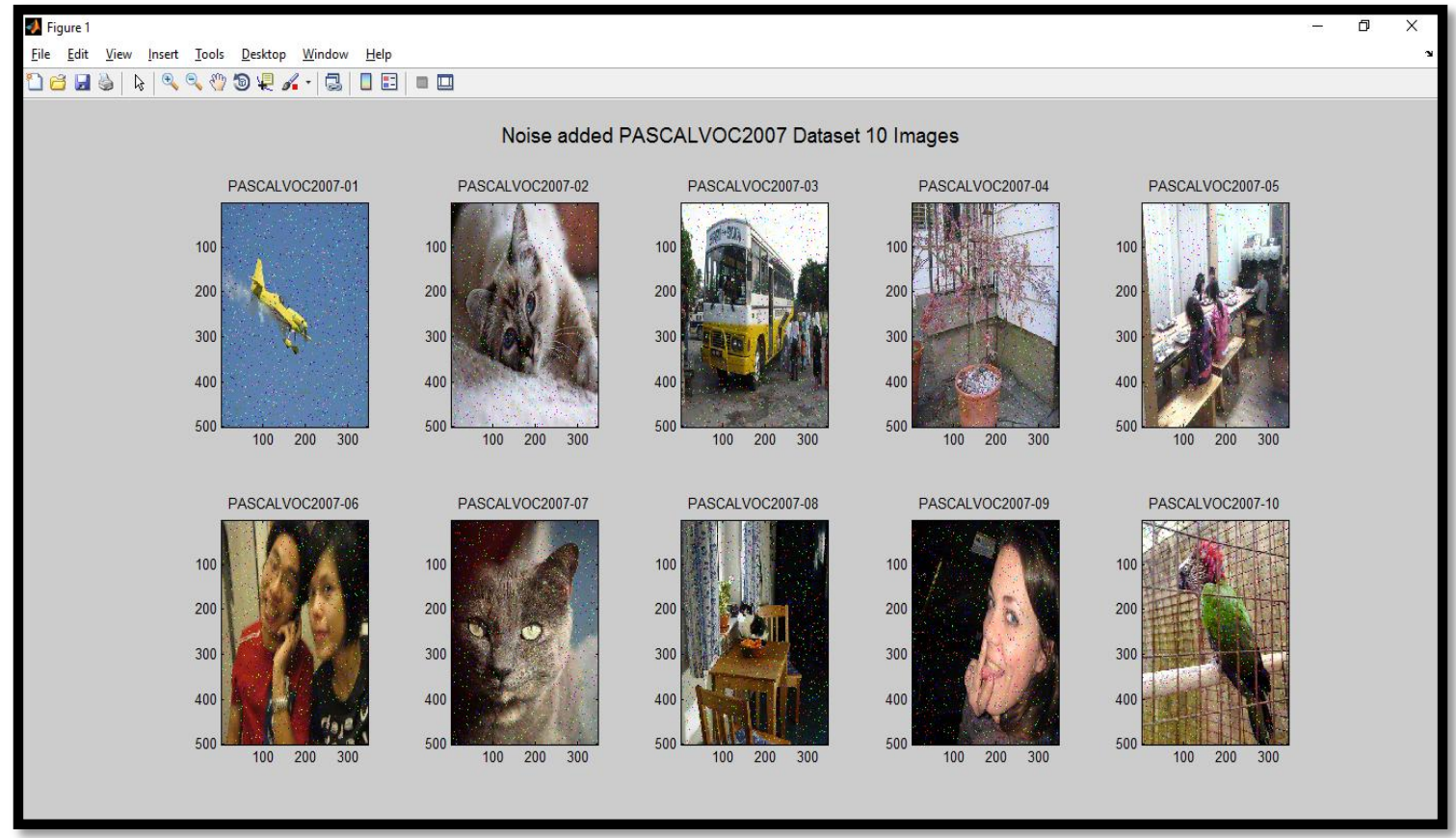

Fig. 10. Noise Added Image of PASCAL - VOC - 2007 Dataset 
International Journal of Advanced Research in Computer and Communication Engineering ISO 3297:2007 Certified

Vol. 6, Issue 3, March 2017

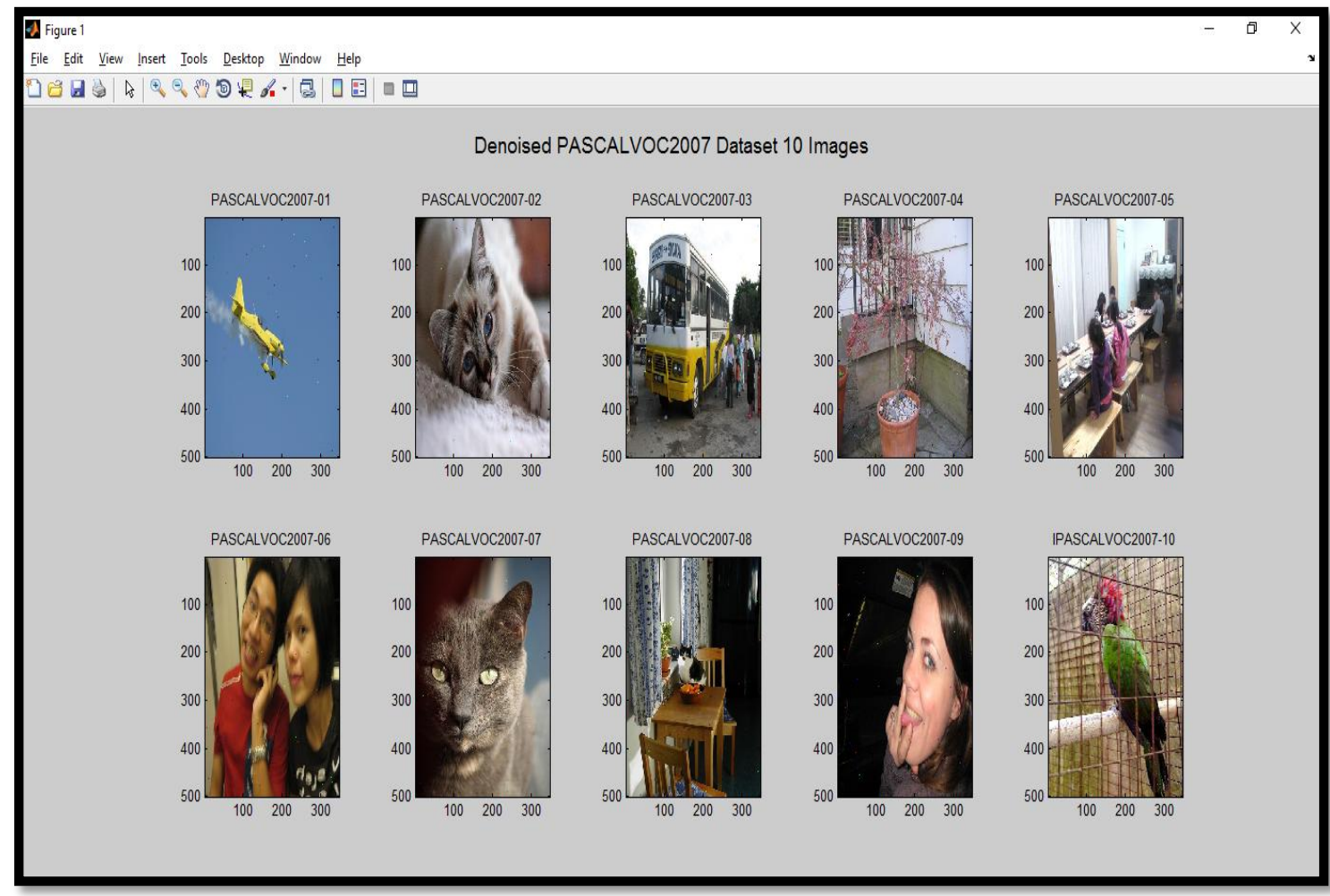

Fig. 11. Denoised Image for PASCAL - VOC - 2007 Dataset

Table 03. PSNR Comparison for the PASCAL - VOC - 2007 Dataset

\begin{tabular}{|c|c|c|c|c|c|}
\hline \multirow{2}{*}{\begin{tabular}{l} 
Image Title \\
\cline { 2 - 6 }
\end{tabular}} & \multicolumn{5}{|c|}{ PSNR in dB } \\
\cline { 2 - 6 } & & & & \\
\hline PASCAL-VOC-2007-01 & 31.7 & 29.2 & 24.3 & 19.7 & 19.4 \\
\hline PASCAL-VOC-2007-02 & 31.3 & 28.3 & 24.4 & 22.8 & 16.9 \\
\hline PASCAL-VOC-2007-03 & 31.1 & 28.7 & 24.4 & 21.9 & 17.9 \\
\hline PASCAL-VOC-2007-04 & 31.0 & 30.5 & 24.2 & 23.6 & 16.1 \\
\hline PASCAL-VOC-2007-05 & 30.7 & 31.4 & 27.3 & 21.7 & 19.0 \\
\hline PASCAL-VOC-2007-06 & 34.1 & 30.1 & 26.9 & 24.1 & 19.7 \\
\hline PASCAL-VOC-2007-07 & 33.5 & 30.6 & 26.1 & 24.7 & 18.0 \\
\hline PASCAL-VOC-2007-08 & 30.3 & 27.0 & 27.8 & 20.8 & 15.7 \\
\hline PASCAL-VOC-2007-09 & 31.5 & 30.4 & 28.0 & 24.2 & 18.6 \\
\hline PASCAL-VOC-2007-10 & 32.2 & 29.5 & 26.9 & 23.2 & 16.4 \\
\hline
\end{tabular}

[where \# $\rightarrow$ L. Rudin et al.,1992, @ $\rightarrow$ Q. Yang et al.,2009, $\& \rightarrow$ T. Qiu et al.,2013, $\rightarrow$ Minyoung Kim.,2015] 


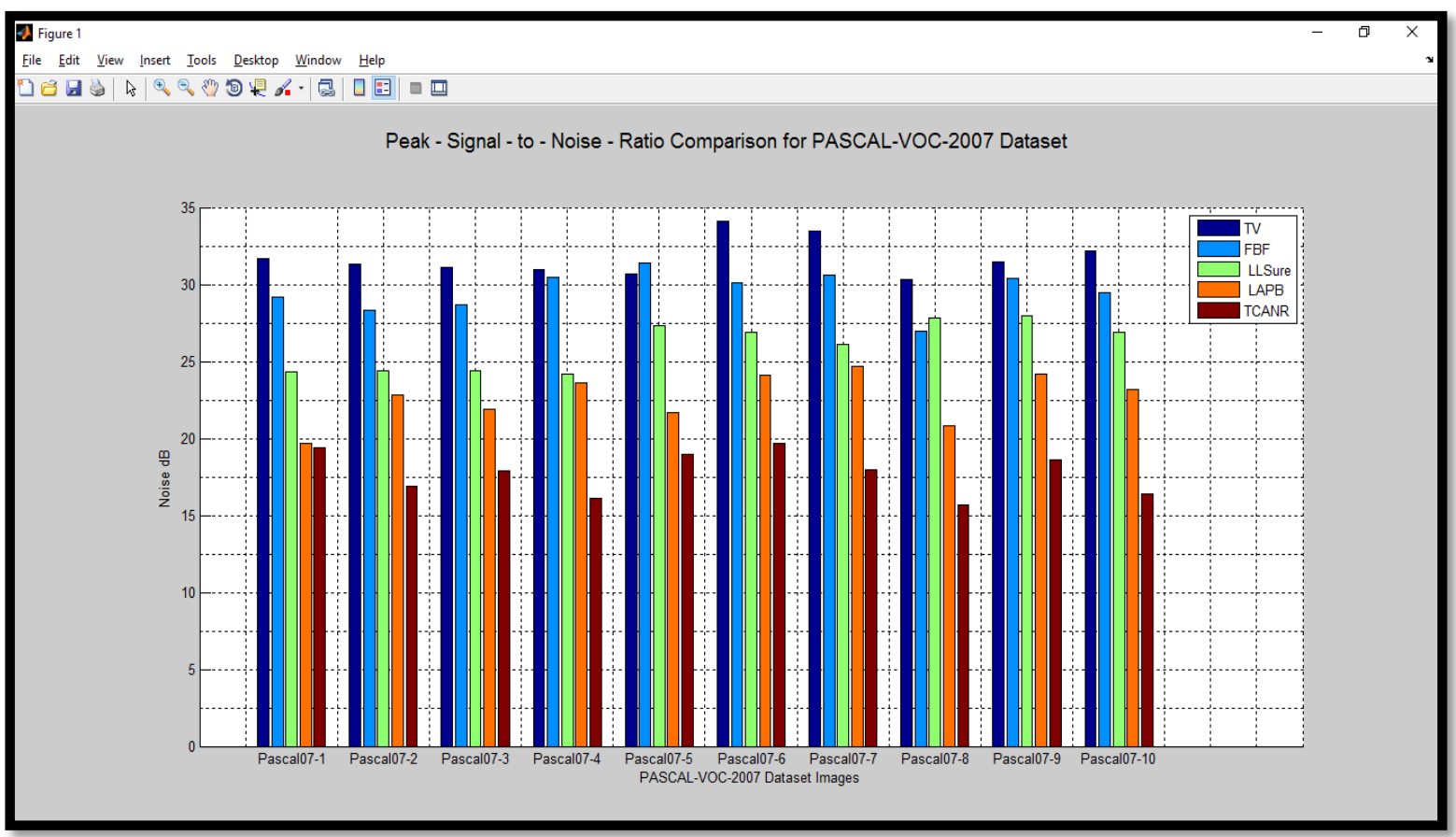

Fig. 12. Matlab Result - PSNR Comparison for PASCAL - VOC - 2007 Dataset

\subsection{PASCAL - VOC - 2010 Dataset}

It is the extension of PASCAL-VOC-2007 dataset with the following developments: (a) Action Classification taster introduced (b) Associated challenge on large scale classification introduced based on ImageNet (c) Amazon Mechanical Turk used for early stages of the annotation.

The obtained original images of PASCAL-VOC-2010 dataset are shown in the Fig.13. Then salt and pepper noise is added to the images of PASCAL-VOC-2010

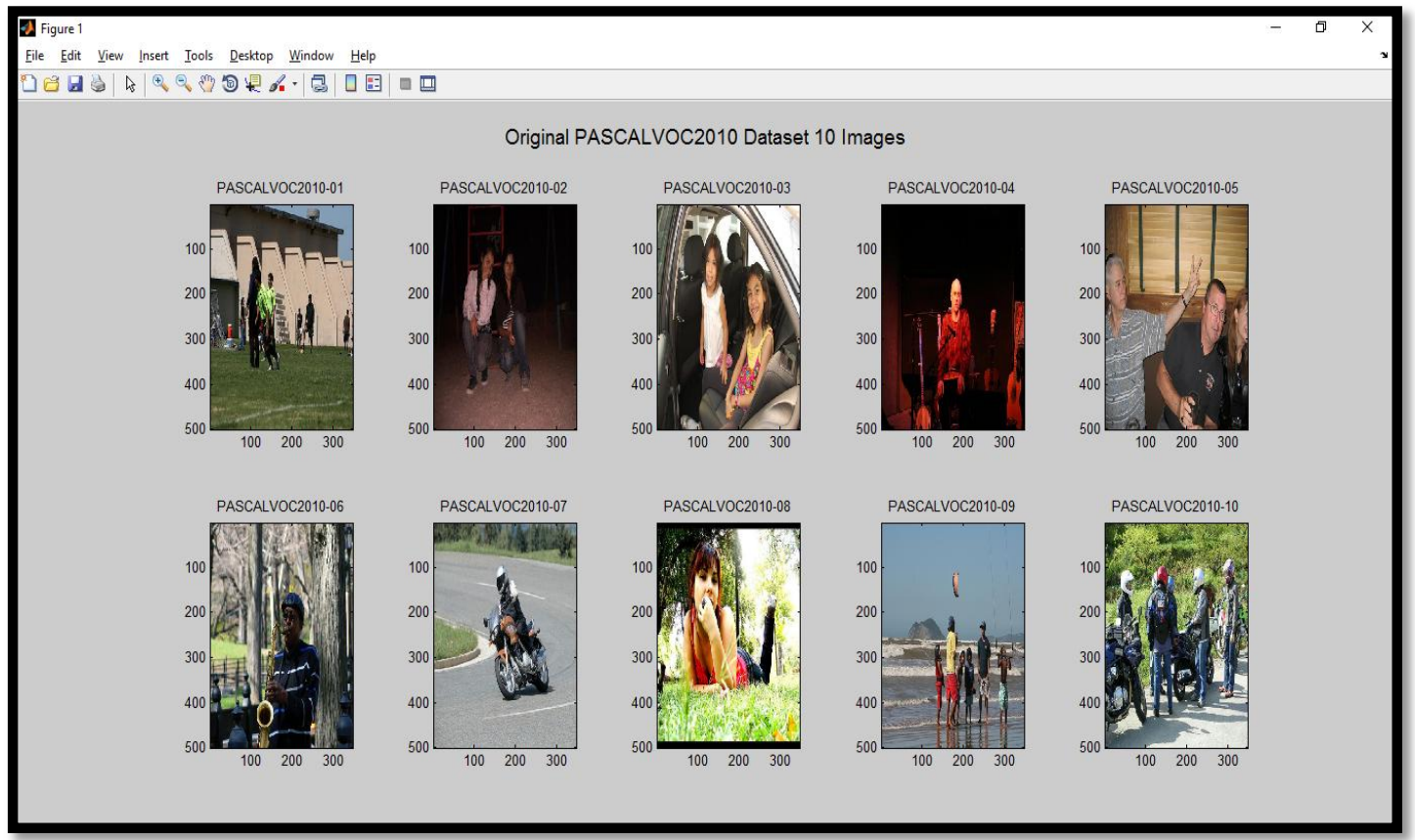

Fig. 13. Original Image of PASCAL - VOC - 2010 Dataset dataset is shown in Fig.14. Then the denoised result image of the proposed TCANR is presented for the PASCALVOC-2010 dataset in Fig. 15. The PSNR comparison for the dataset is presented in the Fig.16. The PSNR values for the each of the four datasets are presented in the Table 4. From the results inferences made is, the maximum noise ratio for the dataset PASCAL-VOC-2010 is $14.9 \mathrm{~dB}$ and the minimum noise ratio is $18.9 \mathrm{~dB}$. This projects the improved performance of TCANR in PASCAL-VOC2010 dataset. 
International Journal of Advanced Research in Computer and Communication Engineering ISO 3297:2007 Certified

Vol. 6, Issue 3, March 2017

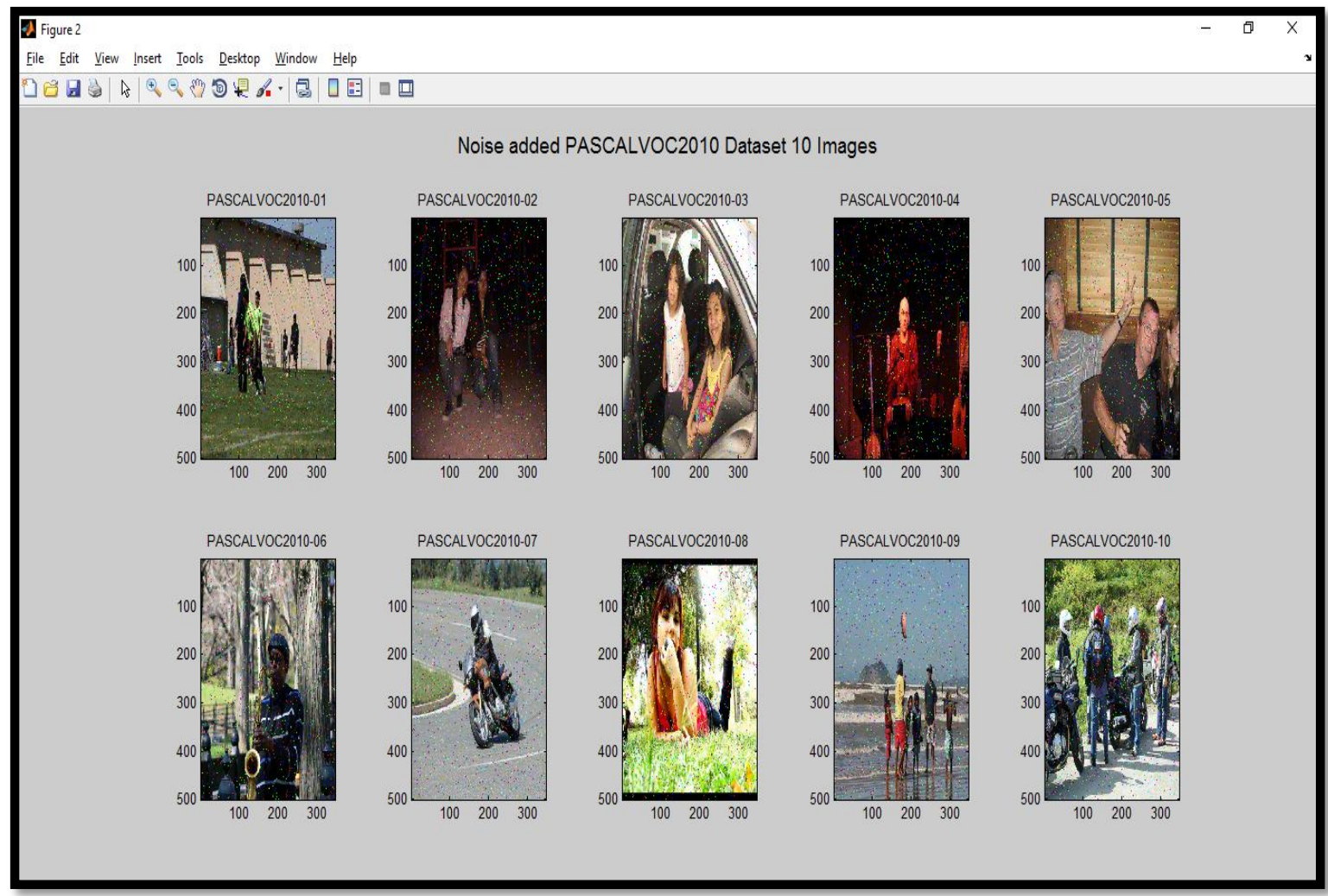

Fig. 14. Noise Added Image of PASCAL - VOC - 2010 Dataset

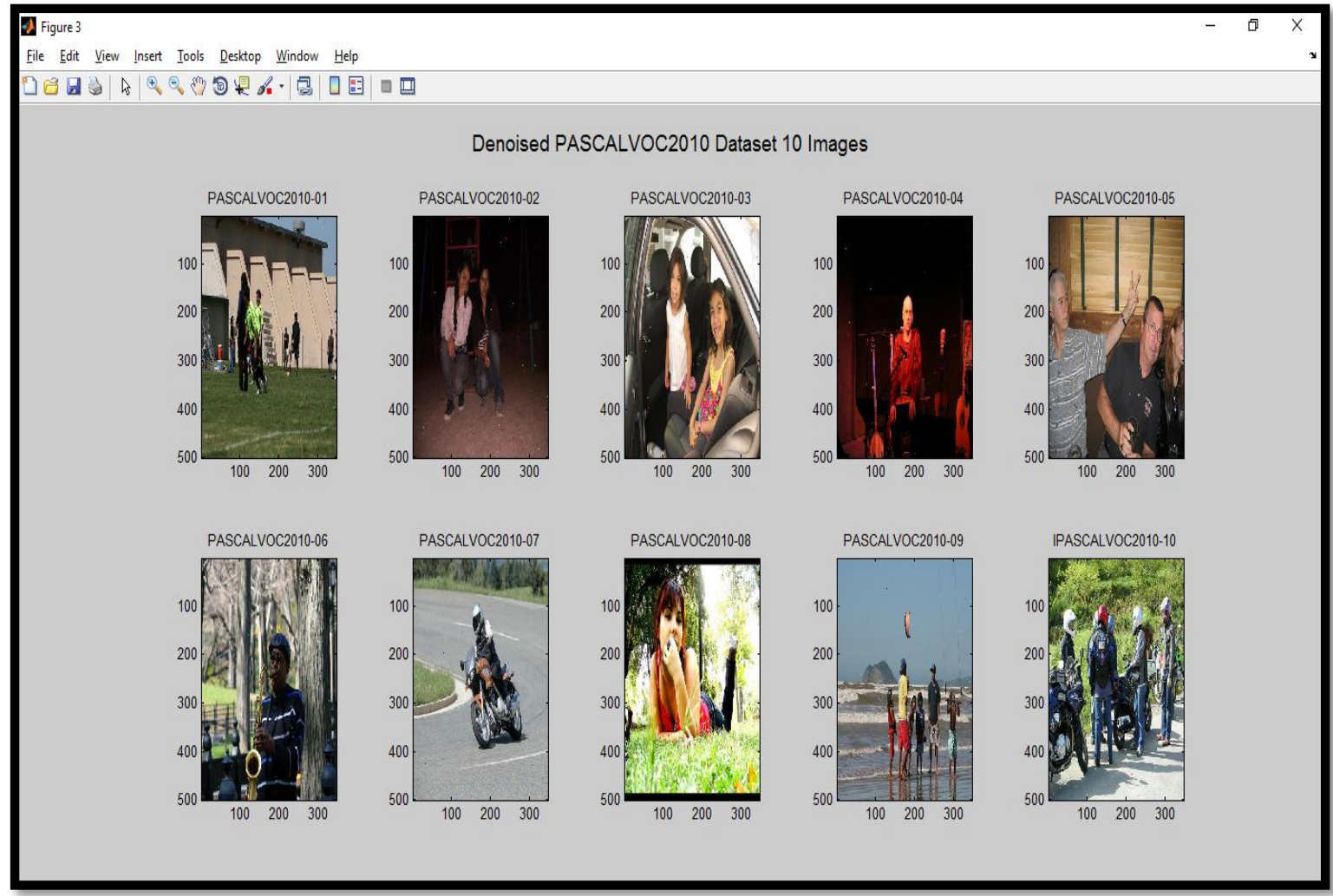

Fig. 15. Denoised Image for PASCAL - VOC - 2010 Dataset 
International Journal of Advanced Research in Computer and Communication Engineering ISO 3297:2007 Certified

Vol. 6, Issue 3, March 2017

Table 04. PSNR Comparison for the PASCAL - VOC - 2010 Dataset

\begin{tabular}{|c|c|c|c|c|c|}
\hline \multirow{2}{*}{ Image Title } & \multicolumn{5}{|c|}{ PSNR in dB } \\
\cline { 2 - 6 } & TV [\#] & FBF [@] & LLSure [\&] & LAPB [*] & TCANR \\
\hline PASCAL-VOC-2010-01 & 30.2 & 29.0 & 24.6 & 21.4 & 16.1 \\
\hline PASCAL-VOC-2010-02 & 30.8 & 26.2 & 27.2 & 22.7 & 15.3 \\
\hline PASCAL-VOC-2010-03 & 31.7 & 27.0 & 27.1 & 19.3 & 18.1 \\
\hline PASCAL-VOC-2010-04 & 33.5 & 26.6 & 27.2 & 19.0 & 17.4 \\
\hline PASCAL-VOC-2010-05 & 33.1 & 29.2 & 27.2 & 18.8 & 18.9 \\
\hline PASCAL-VOC-2010-06 & 29.7 & 30.3 & 23.2 & 23.5 & 14.9 \\
\hline PASCAL-VOC-2010-07 & 31.0 & 28.5 & 24.6 & 22.3 & 16.3 \\
\hline PASCAL-VOC-2010-08 & 31.3 & 29.8 & 24.5 & 19.2 & 17.6 \\
\hline PASCAL-VOC-2010-09 & 33.9 & 27.4 & 22.9 & 23.3 & 16.7 \\
\hline PASCAL-VOC-2010-10 & 29.4 & 30.4 & 27.3 & 19.0 & 17.0 \\
\hline
\end{tabular}

[where \# $\rightarrow$ L. Rudin et al.,1992, @ $\rightarrow$ Q. Yang et al.,2009, \& $\rightarrow$ T. Qiu et al.,2013, $\rightarrow$ Minyoung Kim.,2015]

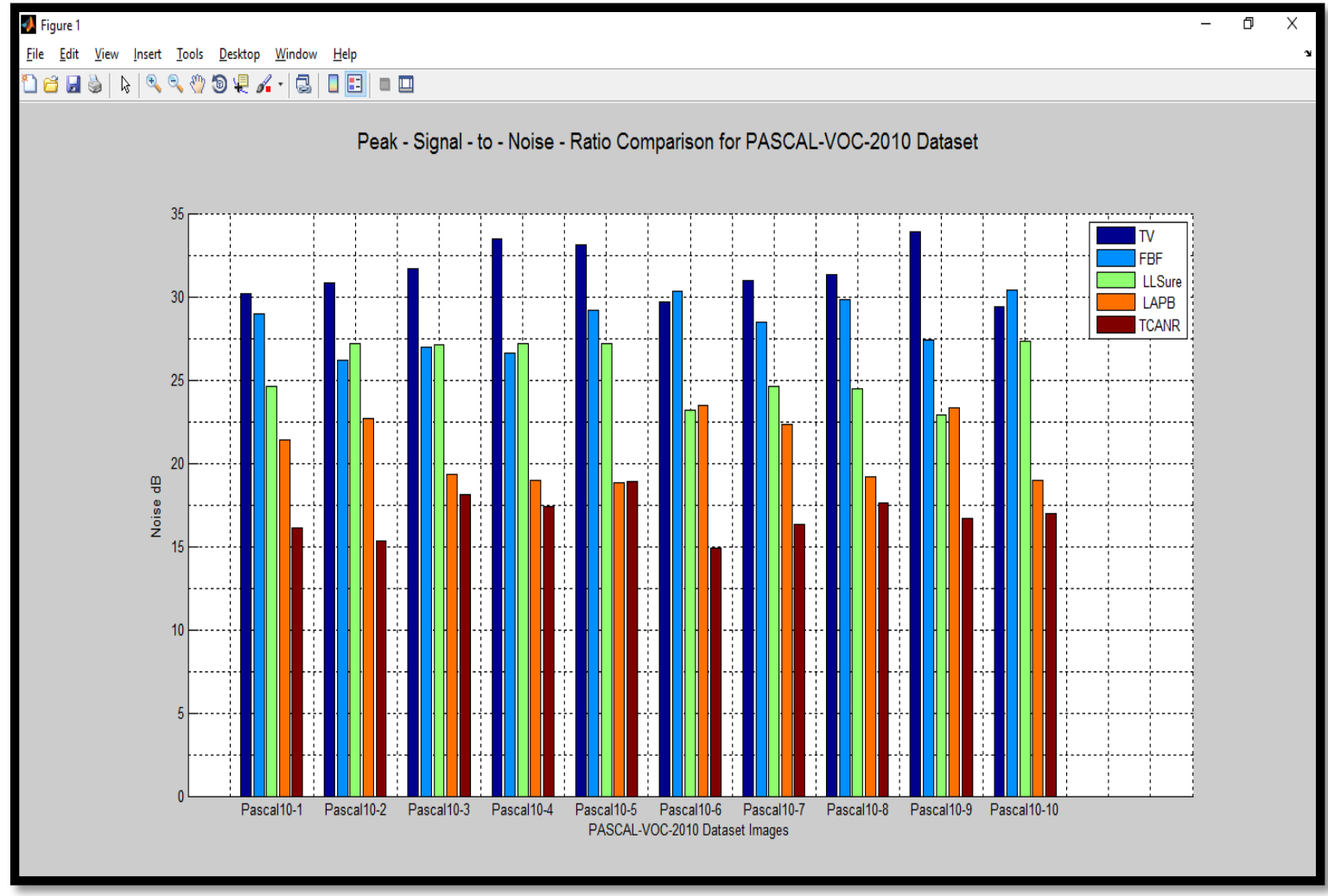

Fig. 16. Matlab Result - PSNR Comparison for PASCAL - VOC - 2010 Dataset

From the results (Fig.4, Fig.8, Fig.12 and Fig.16.) it is evident that the proposed TCANR obtains better performance than the existing methods (TV, FBF, LLSure and LAPB). 


\section{International Journal of Advanced Research in Computer and Communication Engineering} ISO 3297:2007 Certified

Vol. 6, Issue 3, March 2017

\section{CONCLUSIONS}

Weighted Nuclear Norm Minimization makes use of broad-neighbor ascetic-resemblance to concentrate associated area of a given reference into a grid to structure a grouping. Three shortcomings in the existing WNNM method have been identified. In order to overcome these shortcomings Trio Constrained Adaptive Noise Removal (TCANR) is proposed in this research work. The performance of this TCANR is compared with 4 methods (Total Variation (TV) Method, Fast Bilateral Filter (FBF) Method, LL Sure (LLSure) Method, Locally Adaptive Patch - Based (LAPB) Method over four datasets namely Corel 5k Dataset, IAPR - TC12 Dataset, PASCAL-VOC2007 Dataset and PASCAL-VOC-2010 Dataset. Widened simulations are conducted in MATLAB tool and the results affirms that the proposed TCANR performs better in terms of reducing the noise which is measured by PSNR. Future research may be performed by enhancing the proposed TCANR with other noise models.

\section{REFERENCES}

1. Amarjit Roy, Joyeeta Singha, Salam Shuleenda Devi, Rabul Hussain Laskar, Impulse noise removal using SVM classification based fuzzy filter from gray scale images, Signal Processing, Volume 128, November 2016, Pages 262-273.

2. Amarjit Roy, Rabul Hussain Laskar, Multiclass SVM based adaptive filter for removal of high density impulse noise from color images, Applied Soft Computing, Volume 46, September 2016, Pages 816-826.

3. Amarjit Roy, Rabul Hussain Laskar, Non-casual linear prediction based adaptive filter for removal of high density impulse noise from color images, AEU - International Journal of Electronics and Communications, Volume 72, February 2017, Pages 114-124

4. Bin Zhou, Chun-Lai Mu, Jun Feng, Wei Wei, Continuous level anisotropic diffusion for noise removal, Applied Mathematical Modelling, Volume 36, Issue 8, August 2012, Pages 3779-3786.

5. Ching-Ta Lu, Yung-Yue Chen, Ling-Ling Wang, Chun-Fan Chang, Removal of salt-and-pepper noise in corrupted image using threevalues-weighted approach with variable-size window, Pattern Recognition Letters, Volume 80, 1 September 2016, Pages 188199.

6. Guorong Gao, Yanping Liu, Demetrio Labate, A two-stage shearlet-based approach for the removal of random-valued impulse noise in images, Journal of Visual Communication and Image Representation, Volume 32, October 2015, Pages 83-94.

7. Jian Lu, Lixin Shen, Chen Xu, Yuesheng Xu, Multiplicative noise removal in imaging: An exp-model and its fixed-point proximity algorithm, Applied and Computational Harmonic Analysis, Volume 41, Issue 2, September 2016, Pages 518-539.

8. L. Rudin, S. Osher, E. Fatemi, Nonlinear total variation based noise removal algorithms, Physica D 60 (1992) 259-268.

9. Lianghai Jin, Zhiliang Zhu, Xiangyang Xu, Xiang Li, Two-stage quaternion switching vector filter for color impulse noise removal, Signal Processing, Volume 128, November 2016, Pages 171-185.

10. Luis González Jaime, Etienne E. Kerre, Mike Nachtegael, Humberto Bustince, Consensus image method for unknown noise removal, Knowledge-Based Systems, Volume 70, November 2014, Pages 64-77.

11. M. Everingham, L. Van Gool, C.K.I. Williams, J. Winn, A. Zisserman, The PASCAL Visual Object Classes Challenge 2007 (VOC2007) Results <http://www.pascalnetwork.org/challenges/VOC/voc2007/worksho p/index.html>.

12. M. Everingham, L.V. Gool, C.K.I. Williams, J. Winn, A Zisserman, The PASCAL visual object classes (VOC) challenge, Int. J. Comp. Vis. 88 (2) (2010) 303-338.

13. M. Grubinger, Analysis and Evaluation of Visual Information Systems Performance, Ph.D. Thesis, Victoria University, Melbourne, Australia, 2007.
14. Minyoung Kim, Multiple-concept feature generative models for multi-label image classification, Computer Vision and Image Understanding, Volume 136, July 2015, Pages 69-78

15. Muhammad Habib, Ayyaz Hussain, Saqib Rasheed, Mubashir Ali, Adaptive fuzzy inference system based directional median filter for impulse noise removal, AEU - International Journal of Electronics and Communications, Volume 70, Issue 5, May 2016, Pages 689697.

16. P. Duygulu, K. Barnard, N. de Freitas, D. Forsyth, Object recognition as machine translation: learning a lexicon for a fixed image vocabulary, in: European Conference on Computer Vision, 2002.

17. Q. Yang, K.H. Tan, N. Ahuja, Real-time $\mathrm{O}(1)$ bilateral filtering, IN: Proceedings of IEEE Conference on Computer Vision and Pattern Recognition, Miami, FL, 2009, pp. 557-564.

18. Sasan Mahmoodi, Discontinuity preserving method for noise removal of multi-carrier signals, Signal Processing, Volume 131, February 2017, Pages 8-19.

19. T. Qiu, A. Wang, N. Yu, A. Song, LLSURE: local linear sure-based edge-preserving image filtering, IEEE Trans. Image Process. 22 (1) (2013) 80-90

20. V.P. Ananthi, P. Balasubramaniam, A new image denoising method using interval-valued intuitionistic fuzzy sets for the removal of impulse noise, Signal Processing, Volume 121, April 2016, Pages 81-93.

21. Xiang Guo, Yulong Li, Tao Suo, Jin Liang, De-noising of digital image correlation based on stationary wavelet transform, Optics and Lasers in Engineering, Volume 90, March 2017.

22. Xianquan Zhang, Feng Ding, Zhenjun Tang, Chunqiang Yu, Salt and pepper noise removal with image inpainting, AEU International Journal of Electronics and Communications, Volume 69, Issue 1, January 2015, Pages 307-313.

\section{BIOGRAPHIES}

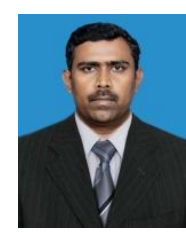

G.Muthukrishnan, done his UnderGraduation in Madurai Kamaraj University and Post-Graduate in Bharathidasan University. He is currently pursuing his Ph.D in Computer Science in Dravaidian University, Kuppam, AndraPradesh. Also he is working as a Senior Project Manager in IT services company. He is having more than one year of research experience and teaching experience as well.

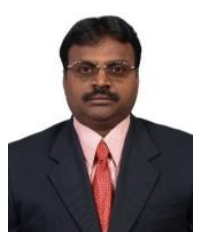

Capt..Dr.S.Santhosh Baboo, aged forty six, has around Twenty Five years of postgraduate teaching experience in Computer Science, which includes Six years of administrative experience. $\mathrm{He}$ is a member, board of studies, in several autonomous colleges, and designs the curriculum of undergraduate and postgraduate programmes. He is a consultant for starting new courses, setting up computer labs, and recruiting lecturers for many colleges. Equipped with a Masters degree in Computer Science and a Doctorate in Computer Science, he is a visiting faculty to IT companies. 